\section{Fortbildungsseminar der APT}

Das 20. Fortbildungsseminar der AG Physik und Technik (APT) der Deutschen Röntgengesellschaft findet in diesem Jahr vom 10. bis 11. Juni in Magdeburg statt.

Schwerpunkte des Seminars sind die Sicherheit im MRT und der Strahlenschutz bei Interventionen. Zudem werden, wie in jedem Jahr, allgemeine Aspekte des Strahlenschutzes behandelt, einschließlich aktueller Änderungen in den dazugehörigen Rechtsvorschriften. Dabei kommt der Etablierung des Medizin-Physik-Expertens natürlich eine besondere Bedeutung zu.

In diesem Jahr werden wir mit einer Reihe von Vorträgen in Kooperation mit ande- ren AGs der Deutschen Röntgengesellschaft starten. Dazu beginnen wir mit einer gemeinsamen Sitzung mit der @GIT zum Thema PACS.

Auch diesmal wird es sicherlich eine schöne Veranstaltung mit abwechslungsreichem Programm und ausreichend Zeit für Diskussionen und persönliche Gespräche mit Kollegen und Referenten werden.

Ich freue mich, Sie in Magdeburg begrüßen zu dürfen und lade Sie ein, auch die wenigen erhaltenen Orte in der „OttoStadt" zu besuchen.

Kerstin Jungnickel

Universitätsklinikum Magdeburg“
Die „Fakten“ zum Seminar

$\nabla$

Datum: 10. - 11. Juni 2016

Tagungsort: Gesellschaftshaus im Klosterbergegarten, Schönebecker Straße 129, 39104 Magdeburg

Wiss. Leitung: Dr. rer. nat. Kerstin Jungnickel

Anmeldung: Online über den Veranstaltungskalender der Akademie für Fortund Weiterbildung in der Radiologie: drg. de $>$ Veranstaltungskalender

Hier finden Sie alle Infos zum Programm: http://www.apt.drg.de/de-DE/2237/20apt-seminar

Ansprechpartner: Svenja Ems (ems@drg. de) und Dr. Martin Völker (voelker@drg. de) 